\title{
ON THE INTENSITY OF CROSSINGS BY A SHOT NOISE PROCESS
}

\author{
TAILEN HSING, ${ }^{*}$ University of Illinois at Urbana-Champaign
}

\begin{abstract}
The crossing intensity of a level by a shot noise process with a monotone response is studied, and it is shown that the intensity can be naturally expressed in terms of a marginal probability.
\end{abstract}

Consider the shot noise process

$$
X(t)=\sum_{\tau \leqq t} h(t-\tau), \quad t \in \mathbb{R},
$$

where the $\tau$ 's are the points of a stationary Poisson process $\eta$ on $\mathbb{R}$ with mean rate $\lambda>0$, and $h$, the impulse response, is a non-negative function on $[0, \infty)$ such that

(i) $h$ is non-increasing,

(ii) $h$ is finite except possibly at 0 ,

(iii) $\int_{u}^{\infty} h(x) d x<\infty$ for some large $u$.

By Theorem 1 of Daley (1971), the conditions (ii) and (iii) ensure that $X(t)<\infty$ almost surely for each $t$.

Observe that the sample function of $X$ increases only at the points of $\eta$. Thus it is clear to define that $X$ upcrosses the level $u$ at $t$, where $u \geqq 0$, if $X(t-) \leqq u$ and $X(t)>u$. For $u \geqq 0$, write $N_{u}$ for the point process (cf. Kallenberg (1976)) that consists of the points at which upcrossings of level $u$ by $X$ occur. Thus for each Borel set $B, N_{u}(B)$ denotes the number of upcrossings of $u$ by $X$ in $B . N_{u}$ is a stationary point process, which may be viewed as a thinned process of $\eta$. The purpose of this communication is to derive the following result.

Theorem 1. For each $u \geqq 0, \mathscr{E} N_{u}(B)=\lambda m(B) P[u-h(0)<X(0) \leqq u]$ for each Borel set $B$, where $m$ is Lebesgue measure.

To prove Theorem 1, first enumerate the points of $\eta$ in $(-\infty, 0)$ by letting $\rho_{i}$ be the $i$ th largest point of $\eta$ to the left of 0 for $i=1,2,3, \cdots$. The $\rho_{i}$ are almost surely well defined, and $-\rho_{1}, \rho_{1}-\rho_{2}, \rho_{2}-\rho_{3}, \cdots$ are independent and identically distributed random variables. The following result is useful.

Lemma 2. For each $i=1,2, \cdots, P\left[X\left(\rho_{i}-\right)=\sum_{j \geqq i+1} h\left(\rho_{i}-\rho_{j}\right)\right]=1$ where $X\left(\rho_{i}-\right)$ denotes the left-hand limit of $X$ at $\rho_{i}$. From this it follows immediately that $X\left(\rho_{i}-\right)$ is independent of $\rho_{i}$, and $X\left(\rho_{i}-\right)$ has the same distribution as $X(0)$.

Received 15 April 1986; revision received 9 December 1986.

* Postal address: Department of Statistics, University of Illinois at Urbana-Champaign, 101 Illini Hall, 725 South Wright Street, Champaign, IL 61820, U.S.A.

Research partially supported by the Air Force Office of Scientific Research Grant No. AFOSR F49620 82 C 0009. 
Proof. Let $i \geqq 1$ be fixed. Since $h$ is monotone, it is almost everywhere continuous. Using the continuity of $\rho_{i}-\rho_{j}, j \geqq i+1$, we obtain

$$
\lim _{\varepsilon \rightarrow 0} h\left(\rho_{i}-\rho_{j}-\varepsilon\right)=h\left(\rho_{i}-\rho_{j}\right) \quad \text { a.s. for } j \geqq i+1 .
$$

Also by the monotonicity of $h, h\left(\rho_{i}-\rho_{j}-\varepsilon\right) \leqq h\left(\rho_{i+1}-\rho_{j}\right)$ for $0<\varepsilon<\rho_{i}-\rho_{i+1}$, $j \geqq i+2$, where $\sum_{j \geqq i+2} h\left(\rho_{i+1}-\rho_{j}\right)$ is almost surely finite since it has the same distribution as $X(0)$. Thus it follows from dominated convergence that almost surely

$$
\lim _{\varepsilon \downarrow 0} X\left(\rho_{i}-\varepsilon\right)=\lim _{\varepsilon \downarrow 0} \sum_{j \geqq i+1} h\left(\rho_{i}-\rho_{j}-\varepsilon\right)=\sum_{j \leq i+1} h\left(\rho_{i}-\rho_{j}\right) .
$$

Proof of Theorem 1. By stationarity, it apparently suffices to show that $N_{u}(B)$ equals $\lambda m(B) P[u-h(0)<X(0) \leqq u]$ for each Borel set $B$ in $(-\infty, 0)$, where $m(B)$ denotes the Lebesgue measure of $B$. Since

$$
X\left(\rho_{i}\right)=h(0)+\sum_{j \geqq i+1} h\left(\rho_{i}-\rho_{j}\right)
$$

Lemma 2 implies that almost surely

$$
N_{u}(B)=\sum_{i \geqq 1} 1\left(u-h(0)<X\left(\rho_{i}-\right) \leqq u, \rho_{i} \in B\right),
$$

where $1(\cdot)$ is the indicator function. Applying the facts that $X\left(\rho_{i}\right)$ is independent of $\rho_{i}$ and $X\left(\rho_{i}-\right)$ is equal in distribution to $X(0)$, we get

$$
\begin{aligned}
\mathscr{E} N_{u}(B) & =\sum_{i \geqq 1} \mathscr{E} 1\left(u-h(0)<X\left(\rho_{i}-\right) \leqq u\right) \mathscr{E} 1\left(\rho_{i} \in B\right) \\
& =P[u-h(0)<X(0) \leqq u] \lambda m(B) .
\end{aligned}
$$

We mention the following for completeness.

(a) By stationarity, the downcrossing intensity of a level by $X$ is also given by Theorem 1.

(b) We assumed, for simplicity of illustration, that the impulse response $h$ is deterministic. Lifting this restriction, it is readily seen that Theorem 1 continues to hold if the impulse responses brought about by the points of $\eta$ are independent of $\eta$, and are independent and identically distributed.

(c) For methods of obtaining the marginal distribution of $X$ see Gilbert and Pollak (1960).

(d) The crossing intensities of some other shot noise processes were studied by Rice (1944), and Bar-David and Nemirovsky (1972). A result in the latter paper can be reduced to one which is similar to Theorem 1 . However, our assumptions on $h$ are considerably simpler.

\section{Acknowledgement}

I am grateful to the referee for his comments.

\section{References}

BAR-DAVID, I. AND NEMIROVSKY, A. (1972) Level crossings of nondifferentiable shot process. IEEE Trans. Inform. Theory 18, 27-34.

DALEY, D. J. (1971) The definition of a multi-dimensional generalization of shot noise. $J$. Appl. Prob. 8, 128-135. 
Gilbert, E. N. ANd Pollak, H. O. (1960) Amplitude distribution of shot noise. Bell System Tech. J. 30, 333-350.

KallenberG, O. (1976) Random Measures. Akademie-Verlag, Berlin: Academic Press, New York.

RiCE, S. O. (1944) Mathematical analysis of random noise. Bell System Tech. J. 24, 46-156. 